\title{
Phénomènes et processus intervenant dans les rapports aux savoirs de la physique: cas d'élèves français en 10 ième année de formation
}

\section{Patrice Venturini}

Cet article concerne l'implication des élèves dans leur travail scolaire en physique. Cette question, qui a donné lieu dans les pays anglo-saxons à de très nombreux travaux sur les attitudes envers les sciences, est ici envisagée en utilisant le rapport au savoir comme cadre théorique. Des travaux réalisés précédemment avaient permis de construire cinq rapports idéal-typiques aux savoirs de la physique, caractérisés par la nature de la mobilisation associée à chacun d'eux. L'étude présentée ici poursuit ce travail et a permis d'identifier une partie des phénomènes et des processus intervenant dans ces rapports aux savoirs. Ces phénomènes concernent l'environnement scolaire, les représentations liées à la physique académique et le projet professionnel. Elle a aussi permis d'examiner l'articulation des rapports aux savoirs de la physique avec les rapports au savoir scolaire.

La question de l'implication de l'élève dans ses activités d'apprentissage est récurrente, mais elle prend une importance particulière dans l'enseignement de la physique, cette discipline souffrant depuis quelques années de désaffection scolaire dans de nombreux pays ${ }^{1}$. La recherche en éducation scientifique l'a abordée essentiellement par l'analyse des attitudes envers les sciences, notamment dans les pays anglo-saxons. En France, la didactique s'est plutôt intéressée aux conditions de transmission des savoirs scientifiques. Dans les études auxquelles elle a donné lieu, à l'image des travaux de Piaget dont elle s'est un moment inspirée, l'élève y est modélisé comme un sujet purement cognitif, toujours prêt, en quelque sorte, à apprendre. Cependant, comme l'analyse Caillot (2001), cette approche a des limites parce qu'elle se heurte à la réalité de l'élève et, pour prendre celle-ci en compte «comme la didactique doit le faire, [...] la question de l'élève, pris dans sa singularité, doit être posée». Pour cela, il propose d'utiliser le concept de rapport au savoir, spécifié aux savoirs de la discipline.

Létude présentée ici s'inscrit dans cette perspective. Elle porte sur le rapport entretenu avec les savoirs de la physique par des élèves français en 10 ième année de formation obligatoire. Dans un premier temps, nous ferons un point sur la question en présentant une très brève synthèse sur les travaux relatifs aux atti- 
tudes et quelques informations ponctuelles sur ceux relatifs à la motivation en sciences. Nous expliciterons ensuite les aspects théoriques et méthodologiques concernant le concept de rapport $\mathrm{au}(\mathrm{x})$ savoir(s), puis la problématique à laquelle répond cette étude. Enfin, nous décrirons la méthodologie utilisée avant d'exposer les résultats obtenus et de les discuter.

\section{Etat de La question}

Le champ de recherche sur les attitudes envers les sciences a donné lieu depuis les années 60-70 à plusieurs centaines d'études et à de nombreuses revues de questions parmi lesquelles on peut signaler celles de Gardner (1975), Schibeci (1984), Osborne, Simon et Collins (2003) ou encore, en langue française, Venturini (2004a). Leurs conclusions sont toutefois assez semblables et font état:

- d'une quasi-absence de référence théorique et d'insuffisances méthodologiques: pour une majorité d'études, seule la présence d'une définition ${ }^{2}$, quand toutefois elle existe, tient lieu de référence théorique. Quelques très rares travaux (par exemple Coll, Dalgety \& Salter, 2002) fondent les recherches sur le modèle du comportement planifié. L'absence de cadres théoriques susceptibles de guider les investigations des chercheurs a des conséquences sur le plan méthodologique, pour lequel la variété des procédures et la multiplicité des questionnaires utilisés sont frappantes. Par ailleurs, la qualité même des questionnaires établis est souvent critiquée (Gardner, 1995).

- d'un consensus sur les résultats: cependant, malgré la variété des méthodologies, certains résultats sont récurrents et font une quasi-unanimité. Ainsi, l'attitude envers les sciences à l'école n'apparaît pas très bonne (Piburn \& Baker, 1993), particulièrement envers la physique (Havard, 1996); elle se dégrade au fur et à mesure que l'élève progresse dans ses études, notamment après son entrée dans l'enseignement secondaire (Hendley, Stables \& Stables, 1996). On peut aussi affirmer d'une part que l'attitude envers les sciences est moins bonne chez les filles que chez les garçons (Weinburgh, 1995), surtout vis-à-vis de la physique, et d'autre part que l'environnement scolaire (et notamment l'enseignant) a beaucoup d'importance sur les attitudes (Ebenezer \& Zoller, 1993). Par ailleurs, les élèves semblent avoir plus d'intérêt pour les cours dans lesquels on met l'accent sur la contextualisation applicative des concepts que pour ceux faisant l'objet d'une approche traditionnelle, dans laquelle la physique est enseignée pour elle-même (Reid \& Skryabina, 2002).

En complément à ces travaux sur les attitudes, on peut aussi signaler quelques rares études sur la motivation en sciences, desquelles il est difficile de dégager une unité en raison de leur faible nombre. On pourrait éventuellement transférer aux sciences les conclusion formulées dans des études prenant appui sur d'autres disciplines, mais chacun des multiples modèles utilisés pour rendre compte de la motivation éclaire la réalité de son point de vue, ce qui rend difficile toute com- 
paraison de résultats ${ }^{3}$. L'éclatement conceptuel est en effet ce qui caractérise le plus ces travaux (Cosnefroy, 2004). On pourra tout de même retenir l'influence du contexte scolaire et notamment de la manière d'enseigner, sur la motivation en sciences (Hanrahan, 1998; Meece, 1991).

\section{Rapport au savoir}

Les propos précédents laissent entrevoir l'intérêt qu'il y aurait à utiliser un autre cadre théorique pour travailler la question de l'implication des élèves dans leurs activités scolaires, notamment en physique. Le rapport au savoir ${ }^{4}$, qui a à voir avec la «mobilisation» des élèves (Charlot, 1997, p. 62) est a priori intéressant à considérer dans cette optique.

\section{Eléments de la théorie du rapport au savoir}

Le concept de rapport au savoir a été introduit par Charlot pour traiter du problème de l'échec scolaire, habituellement explicité en terme de manques, de lacunes, d'insuffisances etc. Pour Charlot, l'échec scolaire ne se réduit pas à des handicaps; c'est aussi et surtout "une expérience que l'élève traverse, et quil interprète» (Charlot, 1997, p. 16), une situation dans laquelle il se trouve au cours de son histoire, "sujet, confronté à la nécessité d'apprendre, et à la présence dans le monde de savoirs de divers types» (Charlot, 1997, p. 35). Etudier un sujet dans cette position, en analysant ses propos, sa conduite, sa situation, son histoire..., c'est étudier son rapport au savoir.

Le rapport au savoir peut être considéré comme «un rapport à des processus (l'acte d'apprendre), à des situations d'apprentissage et à des produits (les savoirs comme compétences acquises et comme objets institutionnels, culturels et sociaux). Il est relation de sens et de valeur: l'individu valorise ou dévalorise les savoirs en fonction du sens qu'il leur confere» (Bautier \& Rochex, 1998, p. 34); du sens et de la valeur donnés à un savoir dépendent la mobilisation de l'élève pour l'apprendre. Charlot (1999b) donne plusieurs autres définitions dont celle-ci, plus descriptive: «le rapport au savoir est l'ensemble (organisé) des relations qu'un sujet humain, donc singulier et social, entretient avec tout ce qui relève de l'apprendre et du savoir: objet, contenu de pensée, activité, relation interpersonnelle, lieu, personne, situation, occasion, obligation etc., liés en quelque façon à l'apprendre et au savoir» (p. 3).

Même si, pour un individu, on peut définir un rapport dominant au savoir, Charlot (1999a) évoque aussi la possibilité de "rapports différents avec les différents types de savoirs", qu'il s'agisse de différentes disciplines, ou de différents savoirs disciplinaires; il s'interroge par exemple (Charlot, 1999b, p. 8-9) sur les conditions de transmission de tel ou tel savoir: "qu'est ce qui dans le rapport d'un élève $a u(x)$ savoir(s) l'aide ou l'entrave pour comprendre ce qu'est un volcan, ce qu'est la foudre, ou encore la théorie de l'évolution (pour le comprendre d'une façon conforme à ce que la science a construit)". 
On peut donc, de la même manière, chercher à savoir en quoi le rapport aux savoirs de la physique constitue un moteur ou un frein, en quoi il est mobilisateur ou non, vis-à-vis de l'apprentissage des savoirs de la discipline. On comprend que ce point de vue intéresse particulièrement la didactique de la physique.

\section{Aspects méthodologiques}

Poser la question du rapport au savoir comme une question de sens oblige à essayer de comprendre l'histoire scolaire et sociale de l'élève, en identifiant les processus qui la rendent intelligible. Ces processus complexes sont constitués d'un ensemble de phénomènes qui interagissent selon une dynamique qu'il faut concevoir en termes de "pluralité constructive» (Charlot, Bautier \& Rochex, 1992, p. 33). Les phénomènes en jeu dans un processus de réussite ou d'échec scolaire sont divers: actions (travailler à l'école, souvent chez soi), relations (apprécier le professeur, faire plaisir à ses parents...), représentations (de l'école, d'une discipline, d'un métier...), mobiles (attentes, demandes, valeurs), psychisme (identifications, projections...), opérations cognitives et langagières (argumenter, catégoriser ...), événements (familiaux, personnels) etc. (op. cit., p. 34). Pour comprendre un processus, il faut d'abord identifier les phénomènes en jeu, les comprendre et tenter ensuite de reconstruire, en les articulant, la dynamique du processus.

Pour cela, deux procédures sont utilisées, le bilan de savoir et l'entretien. Dans la première, les élèves ont à répondre par écrit à la question: "J'ai ... ans. J'ai appris des choses, chez moi, dans la cité, à l'école, ailleurs. Qu'est ce qui est important pour moi dans tout ça? Et maintenant qu'est ce que j'en attends?". Un des postulats de la méthode est que l'élève mentionne les seuls savoirs qui font le plus sens pour lui, puisqu'il ne peut tous les rapporter. On a donc là un outil "permettant de travailler sur du sens, construit et produit par l'élève» (op. cit. p. 134). Aux bilans de savoir s'ajoutent, pour certains élèves, des entretiens approfondis semi-directifs pour traiter de leurs histoires singulières.

Les données, notamment les bilans de savoir, font généralement l'objet de trois analyses différentes (Charlot et al., 1992, p. 40): une analyse qui permet de repérer les thèmes dominants correspondant aux phénomènes évoqués précédemment, une analyse quantitative classique, dans laquelle on cherche à cerner quels apprentissages sont évoqués et par quels groupes d'élèves, et une analyse qualitative des pratiques langagières, la manière de mettre en forme les propos étant reliée au sens que leur donnent les élèves.

Les recherches ont montré que les phénomènes identifiés dans les bilans de savoir apparaissent liés les uns aux autres: quand on en repère certains, on en trouve aussi d'autres presque systématiquement. "On peut ainsi constituer des "constellations d'éléments" et les interpréter comme des ensembles de phénomènes dont l'interaction dynamique constitue un processus» (Charlot et al., 1992, p. 40). Les constellations peuvent donc se comprendre comme des constructions du chercheur rendant compte de cohérences apparues entre des données empi- 
riques, et on peut les présenter sous forme d'idéal-type: «on obtient un idéal-type en accentuant unilatéralement un ou plusieurs points de vue et en enchaînant une multitude de phénomènes donnés isolément, diffus et discrets, que l'on trouve tantôt en grand nombre, tantôt en petit nombre et par endroit pas du tout, qu'on ordonne suivant selon les différents points de vue, choisis unilatéralement, pour former un tableau de pensée homogène» (op. cit., p. 41, citant Weber, 1965).

Le concept de rapport au(x) savoir(s) étant précisé, son intérêt pour la didactique explicité et la manière de le mettre en œuvre décrite, précisons maintenant la problématique de l'étude.

\section{Problématique}

Dans une étude précédente, nous avons caractérisé les rapports entretenus par des étudiants en troisième année d'université avec les savoirs de la physique (Venturini \& Albe, 2002), rapports qui étaient tous dominés par une forte composante utilitaire liée à l'obtention du diplôme. Par contre, la perspective professionnelle d'enseigner la physique, qui correspond aux études réalisées, et qui nécessite donc de bien comprendre cette discipline, apparaît lointaine et n'est pas mobilisatrice. L'étude montrait aussi une relation entre les différents rapports aux savoirs, la maitrise conceptuelle des étudiants et leurs représentations de la Physique académique 5 .

Nous avons ensuite caractérisé les rapports aux savoirs de la physique pour des élèves de l'enseignement secondaire de la 8 ème à la 11 ème année de formation (Venturini, 2005). Pour cela, 414 élèves de 11 classes ont répondu par écrit à un questionnaire de type «bilan de savoir», organisé en trois points: apprentissages en physique que les élèves déclarent avoir effectués; enjeux personnels de ces apprentissages (quelles attentes et quelle importance pour eux); objectifs personnels et degré d'implication en cours de physique. Les réponses ont été découpées en unités de sens ${ }^{6}$, les unités de même type regroupées et comptabilisées au sein d'une même variable. Treize variables ${ }^{7}$ ont été utilisées et un tableau récapitulant l'ensemble des informations pour tous les élèves a été constitué. Ce tableau regroupe donc de fait les résultats d'une analyse thématique des bilans de savoirs et d'une analyse quantitative sur les types de savoirs cités ${ }^{8}$. Le tableau a été analysé avec un logiciel de classification automatique9. Cinq classes ont été constituées, et leurs propriétés ont été établies à l'aide des valeurs significatives des tableaux de contingence des différentes variables. Ces propriétés ont ensuite été idéalisées classe par classe pour construire cinq élèves idéal-typiques ${ }^{10}$ quant au rapport aux savoirs de la physique et à la nature de la mobilisation qui en résulte:

- Idéal-type 1: cet élève cite de manière précise de nombreux savoirs appris, dont il attend de comprendre le monde; la physique est importante pour lui tant pour son utilité dans la poursuite des études que pour les savoirs qu'elle 
apporte; il est impliqué en cours de physique, pour apprendre, expliquer et comprendre les phénomènes.

Son rapport idéal-typique peut se caractériser par une forte mobilisation en physique, une centration sur les savoirs d'abord pour la compréhension du monde qu'ils permettent et aussi pour leur utilité dans la poursuite d'études;

- Idéal-type 2: cet élève cite des savoirs de manière précise mais beaucoup moins que le précédent; la physique est importante pour lui plus pour des raisons stratégiques (utilité dans les études) que pour les savoirs apportés; il est impliqué en cours de physique, pour apprendre et comprendre et par utilité. Son rapport idéal-typique peut se caractériser par une mobilisation en physique, une centration sur les savoirs d'avantage liée à leur utilité stratégique qu'aux explications qu'ils apportent sur le monde;

- Idéal-type 3: cet élève évoque seulement les grandes lignes des savoirs appris (thèmes ou domaines); il vient en cours par utilité, mais ce caractère est moins accentué que dans le cas précédent.

Son rapport idéal-typique est caractérisé par une faible mobilisation en physique, pour des raisons utilitaires peu marquées;

- Idéal-type 4: cet élève attend essentiellement des savoirs appris qu'ils soient utiles, dans la vie ou le métier, et donne pour cela de l'importance à la physique; il vient en cours avec l'intention d'apprendre et est impliqué en classe, sans que cette intention ou cette attitude ne soient réellement efficaces pour ce qui est des savoirs appris, toujours cités de manière très vague.

Son rapport idéal-typique est caractérisé par une mobilisation sur ${ }^{11}$ la physique pour des raisons utilitaires liées au quotidien;

- Idéal-type 5: cet élève ne donne aucune importance aux savoirs de la physique et n'attend rien des savoirs appris; il considère que la physique a peu ou pas d'importance; il vient par obligation aux cours, dans lesquels il est peu ou pas impliqué.

Son rapport idéal-typique est caractérisé par une non-mobilisation sur la physique.

Les phénomènes rassemblés dans les différents idéal-types que nous venons de décrire relèvent essentiellement des actions et des mobiles et sont en nombre trop réduit pour permettre de comprendre les processus qui conduisent aux différentes mobilisations observées. Enrichir les constellations précédentes est donc nécessaire. Pour cela, on peut supposer que des phénomènes relatifs à l'environnement scolaire puissent jouer un rôle. En effet, les travaux sur les attitudes et la motivation en sciences ont montré leur importance dans l'implication scolaire de l'élève, et de plus, l'école constitue pratiquement le seul lieu de rencontre entre l'élève et la physique. On peut aussi supposer, au vu de l'étude que nous avons réalisée à l'université, que ces processus puisse faire intervenir des phénomènes relatifs aux représentations de la Physique académique (fonctionnement, fonction sociale, métiers...) et à l'orientation professionnelle. A vouloir compléter les idéal-types définis, il semble donc légitime de s'interroger sur le rôle des phéno- 
mènes liés aux points précédents, et sur la manière dont ils peuvent interagir pour conduire aux mobilisations observées.

Par ailleurs, on peut penser que la mobilisation sur l'apprentissage des savoirs de la physique ne soit pas totalement déconnectée du cadre plus général de la mobilisation pour apprendre au lycée, sans toutefois que l'on puisse apprécier $a$ priori leurs relations. Eclairer l'articulation entre les rapports aux savoirs de la physique et les rapports au savoir scolaire, comme le suggère Charlot (1999a), constitue donc un enjeu intéressant dans l'optique de mieux comprendre la situation en classe de physique.

C'est à ces questions que tente de répondre notre étude.

\section{Méthodologie}

La méthodologie utilisée pour répondre à ces questions comporte trois étapes: choix des élèves participant à l'étude, recueil d'informations par entretien sur les questions annoncées puis analyse de ces informations.

\section{Choix des élèves}

Compléter des idéal-types déjà définis impose de travailler avec des élèves qui en sont très proches et dont les caractéristiques sont pour la plupart équivalentes à celles de l'échantillon initial. Nous avons choisi de travailler avec des élèves en dixième année de formation, la dernière où, en France, la physique est obligatoire pour tous les élèves. D'une part, la physique fait partie du cursus de ces élèves depuis déjà trois ans, ce qui autorise des propos fondés sur une certaine expérience, d'autre part, en fin d'année, les élèves doivent choisir une orientation scientifique, ou littéraire, ou économique..., ce qui est susceptible de les faire réfléchir au sens de leurs études. Nous avons obtenu la participation de quatre sections réparties sur trois établissements dont deux avaient participé à la recherche précédente. Deux sections étaient réputées avoir un bon niveau, les deux autres un niveau faible. Les 132 élèves concernés sont d'origine sociale moyenne ou aisée.

Pour sélectionner parmi eux ceux qui entretiennent un rapport aux savoirs de la physique proche des idéal-types précédents, nous avons repris le début de la procédure utilisée dans l'étude qui nous a servi à les construire: bilan de savoir identique, analyse des unités de sens, puis comparaison des informations obtenues avec celles caractérisant les idéal-types définis. Trente-six élèves ont ainsi été retenus.

\section{Recueil d'informations par entretien}

Nous avons choisi de recueillir les données complémentaires à l'aide d'un entretien semi-dirigé réalisé pendant une trentaine de minutes avec chacun des élèves sélectionnés. L'entretien a été mené autour des quatre axes suivants. 


\section{Confirmer le rapport aux savoirs de la physique}

La première série de questions visait à confirmer, pour l'élève sélectionné, l'existence d'une forte proximité entre le rapport entretenu avec les savoirs de la physique et un des rapports idéal-typiques définis. Pour cela, les élèves ont eu à préciser la nature de leurs relations avec la physique et en expliquer les causes, à décrire leurs pratiques d'étude tant en physique que dans d'autres disciplines ainsi que les raisons qui expliquent leur degré de mobilisation; ils ont eu aussi à mentionner leurs activités extrascolaires impliquant la physique et à apprécier la pertinence de la physique pour comprendre le monde qui les entoure.

\section{Caractériser le rapport au savoir scolaire}

La caractérisation du rapport entretenu par les élèves avec le savoir scolaire a été réalisée à partir

- de questions posées au cours de l'entretien, relatives aux domaines pour lesquels ces lycéens manifestent de l'intérêt, ou qui leur paraissent importants à maîtriser;

- de trois questions auxquelles ils ont eu préalablement à répondre par écrit: "pour quoi, avec quelles intentions venez-vous au lycée?"; "Qu'avez-vous appris d'important depuis que vous allez à l'école?»; "Qu'attendez-vous des savoirs appris?".

\section{Identifier les représentations sur la Physique académique et sur son utilité sociale, préciser la nature du projet professionnel}

Les élèves ont décrit ce qu'ils percevaient du fonctionnement de la Physique académique, des démarches et des outils utilisés par les physiciens, de sa fonction sociale et des métiers associés. Enfin, ils ont caractérisé en quelques mots la Physique dans la société, puis ont explicité leur projet professionnel (métier ou domaine d'activité) ainsi que les raisons de ce choix.

\section{Identifier la perception d'une partie du contexte scolaire relatif à la physique} La suite de l'entretien a conduit les élèves à:

- apprécier globalement l'enseignement de la discipline;

- se positionner sur certaines composantes de cet enseignement liées à la présence d'aspects applicatifs dans les cours, au caractère scolaire ou non des savoirs appris, et aux réponses apportées ou non en cours à leurs interrogations sur le monde;

- définir ce qu'est, selon eux, un bon professeur;

- faire état du degré de réussite scolaire dans cette discipline.

\section{Analyse des informations recueillies}

Les entretiens ont été retranscrits en intégralité puis analysés manuellement à deux reprises, en cherchant à identifier les phénomènes évoqués et leurs modalités. Le traitement a d'abord été effectué globalement pour chacun des individus 
puis il a été repris " transversalement " pour chacun des axes de l'entretien, afin d'homogénéiser la prise en compte des réponses des différents élèves. Ces informations ont été récapitulées dans un tableau qui nous a permis d'effectuer des contrôles de cohérence et de repérer les régularités existantes pour poursuivre la construction des différents idéal-types. Si un phénomène apparaît avec la même modalité dans les propos d'une majorité nette d'élèves associés à un idéal-type, celui-ci est pris en compte. Dans le cas contraire, il est ignoré.

Le rapport au savoir scolaire a été analysé d'une part en repérant dans les bilans écrits les savoirs cités ainsi que les attentes et les intentions exprimées ${ }^{12}$, d'autre part en repérant dans l'entretien, les centres d'intérêt et les pratiques d'études dans différentes disciplines. Les rapports idéal-typiques ont été construits progressivement de manière inductive, en généralisant les informations majoritairement présentes.

Par ailleurs, les réponses fournies en entretien nous ont conduit à éliminer de l'échantillon deux élèves entretenant avec les savoirs de la physique un rapport dont les caractéristiques s'étaient éloignées, entre le questionnaire et l'entretien, des idéal-types de référence. Les résultats que nous allons maintenant donner ont donc été obtenus à partir de 34 entretiens avec des élèves ainsi répartis ${ }^{13}$ :

Tableau 1. Répartition des 34 élèves de l'échantillon selon le rapport entretenu avec les savoirs de la physique

\begin{tabular}{|c|c|}
\hline $\begin{array}{c}\text { Rapport proche de } \\
\text { l'idéal-type }\end{array}$ & Nombre d'élèves \\
\hline 1 & 5 \\
\hline 2 & 8 \\
\hline 3 & 8 \\
\hline 4 & 4 \\
\hline 5 & 9 \\
\hline
\end{tabular}

\section{Résultats}

Nous évoquerons d'abord les compléments apportés aux différents idéal-types et nous préciserons ensuite les articulations entre rapport au savoir scolaire et rapport aux savoirs de la physique.

\section{Caractéristiques des différents idéal-types}

L'exploitation des entretiens permet de compléter ainsi les caractéristiques des différents élèves idéal-typiques quant à leur rapport aux savoirs de la physique:

- Idéal-type 1, dont le rapport aux savoirs peut se caractériser par une forte mobilisation en physique, une centration sur les savoirs pour la compréhension 
du monde qu'ils permettent et l'utilité qu'ils présentent pour les études: cet élève attribue peu d'importance à l'enseignant auquel ni son intérêt ni son travail en physique ne sont liés. Il identifie dans les cours des phénomènes et des applications liés au quotidien, et réutilise à l'extérieur de l'école les savoirs appris en physique. Il est d'ailleurs satisfait de manière générale de l'enseignement de la physique. Il aimerait toutefois obtenir d'avantage de réponses à ses interrogations personnelles. S'il a une représentation évoluée du fonctionnement de la Physique académique et de sa fonction sociale, il n'y attribue pas beaucoup d'importance, contrairement à son projet professionnel dans lequel la physique a une place prépondérante. Il a le sentiment de bien réussir en physique.

$\mathrm{Si}$, à ce stade, on ne peut qu' imaginer les processus au sein desquels s'articulent ces phénomènes, on peut tout de même remarquer que leurs effets se renforcent les uns les autres (par exemple utilisation de savoirs à l'extérieur de l'école et attente que les savoirs permettent de comprendre le monde; réussite scolaire en physique, utilité des savoirs pour les études, et choix professionnel etc.). On peut donc penser qu'ils conduisent à une mobilisation bâtie autour des savoirs de la physique qui a toute les chances de rester stable dans le temps;

- Idéal-type 2, dont le rapport aux savoirs peut se caractériser par une mobilisation en physique, une centration sur les savoirs plus liée à leur utilité stratégique qu'aux explications qu'ils apportent sur le monde: l'importance de l'enseignant est faible pour cet élève dont l'activité en physique résulte d'un choix personnel. Il souhaite des cours incluant beaucoup plus de phénomènes du quotidien pour y trouver de l'intérêt, et estime que les savoirs appris sont peu ou pas utilisables à l'extérieur de l'école, mais ce dernier aspect est cependant peu important pour lui. Les questions qu'il se pose, liées au cours, trouvent des réponses et il a le sentiment de réussir en physique. Il ignore tout du fonctionnement de la Physique académique et de sa fonction sociale, mais n'y attribue pas d'importance. Il a un projet professionnel qui nécessite des études incluant momentanément la physique.

Celle-ci apparait comme une discipline très formelle (pas d'utilisation des savoirs et pas de représentations de la discipline autres que scolaires); les processus concourant à la mobilisation puisent leurs sources pour la plupart à l'extérieur de la physique (réussir les études pour un métier duquel la physique sera absente), et on peut donc penser qu'ils sont conjoncturels;

- Idéal-type 3, dont le rapport aux savoirs peut se caractériser par une faible mobilisation en physique, pour des raisons utilitaires peu marquées: cet élève attribue beaucoup d'importance à l'enseignant sur lequel il compte pour faire naître un intérêt pour la physique. Il n’identifie pas la présence de phénomènes du quotidien dans les cours et trouve que les savoirs appris sont inutilisables à l'extérieur de l'école, ce qui contribue à le démobiliser. Il ignore le fonctionnement de la Physique académique et sa fonction sociale. Il n'a pas de projet professionnel impliquant des études en physique. 
La physique est, sur tous les plans, étrangère à cet élève et, en l'absence d'un enseignant charismatique, les processus que l'on peut imaginer à partir des phénomènes précédents ne sont pas mobilisateurs;

- Idéal-type 4, dont le rapport au savoir peut se caractériser par une mobilisation sur la physique pour des raisons utilitaires liées au quotidien: l'enseignant a beaucoup d'importance pour cet élève ${ }^{14}$. Celui-ci trouve que les cours comportent suffisamment d'exemples concrets, et que les savoirs appris ne sont pas purement scolaires. Il ignore le fonctionnement de la Physique académique et sa fonction sociale mais n'y attribue pas d'importance. Son projet professionnel inclue en partie ou à la marge des connaissances de la physique.

Cet élève trouve dans la vie quotidienne ou dans son projet professionnel un sens à la physique, et des raisons pour se mobiliser. Mais comme le font remarquer Charlot et al. (1992, p. 28), la question du sens n'annule pas celle de l'efficacité des actions: la mobilisation de cet élève, qui ce cite pas de savoirs appris dans son bilan, ou alors de manière très vague, semble plutôt relever du discours ou de l'attitude volontariste, d'où probablement la forte importance attribuée à l'enseignant, qui peut conduire l'élève à mieux réussir.

- Idéal-type 5, caractérisé par une non-mobilisation sur la physique: cet élève attribue une forte importance à l'enseignant dont la passion et les qualités pédagogiques pourraient lui faire aimer et comprendre la physique. L'enseignement de la physique, qu'il trouve complexe, abstrait, ennuyeux et trop rapide ne lui convient pas. Il n'identifie pas dans les cours des explications relatives à des phénomènes du quotidien et pour lui, les savoirs qu'il apprend ne sont pas exploitables à l'extérieur de l'école, ce qui enlève tout intérêt à la discipline. Il ignore le fonctionnement de la Physique académique et sa fonction sociale, et il pense qu'il est ainsi privé d'une source d'intérêt. Il n'a pas de question personnelle qui relève de cette discipline. Son projet professionnel n'implique ni la physique, ni les études en physique, vis-à-vis desquelles il a le sentiment d'être en échec.

En dehors de l'action hypothétique de l'enseignant, tous les autres phénomènes évoqués concourent, quelle que soit la manière dont on imagine leur interaction, à une non-mobilisation de cet élève (par exemple savoirs non opérationnels, échec scolaire, savoirs inutiles professionnellement etc.); celleci a de ce fait toutes les chances de rester stable dans le temps.

\section{Articulation entre rapport au savoir scolaire et rapport aux savoirs de la physique}

L'analyse des propos des élèves dans les bilans de savoir scolaire et dans l'entretien nous a conduit à construire trois élèves idéal-typiques en fonction de leur rapport au savoir scolaire:

- Idéal-type 1, caractérisé par une forte mobilisation sur le savoir scolaire: cet élève a dans la vie des centres d'intérêts clairement identifiés dont la plupart 
sont en relation avec les savoirs scolaires et leur donnent du sens. Il témoigne du désir et du plaisir d'apprendre à l'école afin de satisfaire sa curiosité. Il mentionne dans les savoirs appris des compétences, des savoirs réflexifs liés au raisonnement et à l'esprit critique. Il vient au lycée, dans lequel il réussit bien, pour acquérir des connaissances et des compétences. Il attend des savoirs appris de comprendre le monde, de s'y intégrer personnellement et socialement, notamment par l'autonomie, le développement personnel et culturel et les longues études qu'ils permettent.

Ces phénomènes concernent en profondeur l'individu et sa construction. Les interactions que l'on peut imaginer entre eux, dont les effets se renforcent mutuellement (par exemple plaisir d'apprendre pour satisfaire la curiosité, réussite scolaire, rôle des savoirs dans le développement personnel et culturel etc.), conduisent à une mobilisation au ${ }^{15}$ lycée vraisemblablement durable et efficace.

- Idéal-type 2, caractérisé par une mobilisation moyenne et conjoncturelle sur les savoirs scolaires: cet élève a de la curiosité pour une ou plusieurs disciplines, mais pas au point de les approfondir. Il a appris à l'école les savoirs de base, c'est-à-dire "lire écrire compter» et "les bases de chaque matière», ainsi que des savoirs relationnels. Il classe les savoirs appris selon leur utilité vis-à-vis de la vie ou du projet professionnel, et à ce titre, il juge qu'une large partie des savoirs proposés par l'école est inutile. Il vient au lycée pour «avoir le bac» afin de continuer son chemin dans le cursus scolaire, et pour avoir des relations sociales. Il attend des savoirs appris un diplôme pour pouvoir choisir un bon métier, des connaissances utiles et une intégration dans la société.

On peut imaginer que les processus combinant ces phénomènes puissent conduire à une mobilisation scolaire temporaire et ciblée sur l'acquisition de certains savoirs jugés stratégiquement utiles pour acquérir un diplôme et avoir un statut reconnu dans la société, mais ils ne concernent pas la transformation de l'individu lui-même: à ce titre, la portée de la mobilisation vis-à-vis de l'ensemble des apprentissages scolaires est réduite;

- Idéal-type 3, caractérisé par une très faible mobilisation sur les savoirs scolaires: cet élève n'a pas de centre d'intérêt clairement identifié, il ne sait pas trop ce qu'il aime apprendre ou comprendre à l'école. Il retient des savoirs appris les savoirs de base de l'école primaire (lire écrire compter) et surtout les savoirs relationnels, qui ont le plus d'importance. Même si sa scolarité est difficile, il vient au lycée pour voir ses amis, accéder à un métier et assurer son avenir, l'un et l'autre restant vagues. Il attend des savoirs appris de réaliser ce qu'il désire.

Rien dans cet ensemble de phénomènes ne concourt à finaliser d'éventuels apprentissages (absence d'intérêt identifié, absence de réussite scolaire...), hormis le sentiment quasi-magique que la scolarité au lycée conduit à obtenir ce qu'on désire dans la vie. On comprend donc que les processus ${ }^{16}$ au sein desquels ces phénomènes interagissent ne peuvent conduire à une réelle mobilisation sur les savoirs scolaires. 
Chacun des 34 élèves peut être associé à deux idéal-types dont il est proche, l'un lié au rapport au savoir scolaire, l'autre lié au rapport aux savoirs de la physique; le tableau suivant précise la répartition des effectifs.

Tableau 2. Répartition des élèves de l'échantillon selon les deux idéal-types auxquels ils sont associés.

\begin{tabular}{|c|c|c|c|}
\hline $\begin{array}{c}\text { Idéal-types liés aux rapports : } \\
\text {-au savoir scolaire } \rightarrow \\
\text {-aux savoirs de la physique } \quad \downarrow\end{array}$ & 1 & 2 & 3 \\
\hline 1 & 5 & & \\
\hline 2 & 1 & 7 & \\
\hline 3 & & 4 & \\
\hline 4 & & 4 & 5 \\
\hline 5 & & & \\
\hline
\end{tabular}

La lecture du tableau permet de constater l'existence de liens entre les mobilisations extrêmes. Ainsi, tous les élèves très faiblement mobilisés sur le savoir scolaire ne sont pas mobilisés sur les savoirs de la physique et on peut donc penser que cette non-mobilisation a aussi des sources extérieures à la physique. A l'inverse, tous les élèves fortement mobilisés en physique sont aussi fortement mobilisés sur le savoir scolaire, ce qui constitue tout de même une spécificité importante. Une constatation identique a d'ailleurs été faite sur une population plus large de trois classes en 9 ième année de formation (Venturini, 2004b). Quant aux élèves mobilisés moyennement et conjoncturellement sur le savoir scolaire, ils sont associés, de manière un peu prévisible, à une grande variété d'idéal-types relatifs aux savoirs de la physique: la nature de leur mobilisation vis-à-vis de la physique peut être reliée à la nature du projet professionnel, selon les propos avancés par ces élèves pour justifier leur positionnement. Si les rapports au savoir scolaire et aux savoirs de la physique sont articulés l'un à l'autre, ils ne sauraient donc être confondus.

\section{Discussion}

L'étude précédente (Venturini, 2005) avait permis de construire cinq rapports idéal-typiques entretenus avec les savoirs de la physique par des élèves de l'enseignement secondaire, caractérisés par la nature de la mobilisation associée. L'étude présentée ici, centrée sur des élèves en dixième année de formation, précise une partie des phénomènes à l'origine de ces rapports aux savoirs et permet d'imaginer les processus dans lesquels ils interagissent pour y conduire. A ce titre, 
elle apporte donc un complément d'informations intéressant, notamment par rapport aux travaux sur les attitudes envers les sciences, même si en première analyse, les éléments mis en jeu (par exemple le rôle d'une partie de l'environnement scolaire) apparaissent semblables. En effet, on envisage ici une dynamique d'interaction entre différents phénomènes, différenciée selon des types idéalisés d'élèves, là où on étudiait l'influence séparée de différents facteurs, statistiquement mesurée sur la globalité d'une population indifférenciée.

Cependant, le complément apporté reste partiel à plusieurs titres. L'étude concerne d'abord une tranche d'âge particulière, celle au cours de laquelle les élèves font, en France, un choix d'orientation qu'elle permet certainement d'éclairer. Mais la question de savoir comment on en arrive là se pose aussitôt, et l'examen dans le temps des phénomènes et des processus à l'œuvre s'impose pour comprendre l'évolution qui conduit à la situation constatée en dixième année de formation. Par ailleurs, la population étudiée ne comporte pas, en proportion significative, d'élèves issus des classes sociales défavorisées. Même si, comme on l'a vu, celles-ci n'ont pas l'apanage de la présence d'élèves peu ou pas mobilisés à l'égard du savoir scolaire ou en rupture avec l'étude de la physique, on peut imaginer dans ce contexte, l'existence et l'importance de phénomènes différents. De plus, l'échantillon étudié, même s'il est soigneusement sélectionné, reste réduit. Enfin, même dans le cadre choisi pour cette étude, on peut imaginer, au vu des éléments identifiés dans les travaux sur les attitudes ou la motivation envers les sciences, l'existence d'autres phénomènes. La question du genre, par exemple, régulièrement pointée dans de nombreuses études, laisse supposer que les phénomènes qui y sont liés sont importants. Nous avions d'ailleurs prévu de l'examiner dans les entretiens, mais la méthodologie utilisée n'a pas permis de conclure: les élèves, s'ils attribuaient aux stéréotypes habituels une certaine validité, ne leur accordaient sur eux aucun impact ${ }^{17}$. D'autres phénomènes, liés à l'environnement scolaire, devraient aussi être explorés: nature du curriculum, nature et organisation des activités, objets et procédés d'évaluation etc.

Quant aux rapports au savoir scolaire, les idéal-types que nous avons construits de manière inductive recoupent ceux que l'on peut construire à partir des travaux de Bautier et Rochex (1998, p. 39-44; 123-136), ou ceux qui ont été identifiés par Catel, Coquidé et Gallezot ${ }^{18}$ (2002). Cependant nos idéal-types sont un peu moins riches: d'une part, l'échantillon et le corpus à analyser sont ici nettement plus réduits, et d'autre part, il s'agit de rapport au savoir scolaire et non pas de rapport au savoir. Cette partie de l'étude a cependant permis de constater que le rapport au savoir scolaire ne conditionnait qu'en partie l'apprentissage de la physique ce qui justifie a posteriori l'intérêt qu'il y a à considérer spécifiquement un rapport aux savoirs de la physique. 


\section{Conclusion}

Nous retiendrons tout d'abord de cette étude l'intérêt, pointé dans la discussion, que présente le concept de rapport au savoir pour prolonger et enrichir les recherches sur l'implication des élèves dans le travail scolaire, menées à partir des attitudes envers les sciences et de la motivation en science.

Au-delà des constellations élaborées, présentées sous forme d'idéal-type, qui constituent l'objet essentiel de ce travail, et sur lesquelles nous ne reviendrons pas ici, on peut aussi souligner, dans les résultats précédents:

- l'existence de rapports extrêmes (idéal-type 1 et 5) qui semblent devoir être stables dans le temps;

- la forte importance de l'enseignant dans les idéal-types 3 à 5, sur lequel reposent les espoirs des élèves pour palier l'absence d'une mobilisation personnelle et efficace pour apprendre la physique;

- le faible pouvoir mobilisateur de la physique qui implique à cet âge les seuls élèves concernés par un projet professionnel en relation avec elle ${ }^{19}$;

- la méconnaissance par une majorité d'élève du fonctionnement de la Physique académique, de sa fonction sociale et des métiers qui y sont associés, sans d'ailleurs que celle-ci semble constituer un obstacle au travail scolaire;

- l'articulation entre rapport au savoir scolaire et rapport aux savoirs de la physique, qui semble notamment montrer que les élèves fortement mobilisés en physique sont des élèves fortement mobilisés sur le savoir scolaire.

Enfin, si la piste explorée dans cette étude semble intéressante, il convient de la poursuivre pour en évaluer complètement la portée, de nombreux phénomènes restant vraisemblablement à prendre en compte pour enrichir la connaissance des processus qui concourent à établir le rapport entretenu par des élèves avec les savoirs de la physique, et à le faire évoluer.

\section{Notes}

1 On peut consulter par exemple le rapport Porchet (2002) pour la situation en France.

2 Une définition souvent citée est empruntée à Oppenheim (1992, p. 174): une attitude est «une disposition, une tendance à répondre d'une certaine manière à certains stimuli».

3 Les travaux sur la motivation ont plus pour objectif de tester l'influence de certains facteurs sur la motivation comme état psychologique, c'est-à-dire de valider un modèle, que d'étudier la motivation dans une discipline donnée en fonction de sa spécificité.

4 Je considère ici la théorisation du rapport au savoir relevant de l'approche socio-anthropologique construite par B. Charlot, E. Bautier et J.-Y. Rochex.

5 Le terme "Physique " est écrit avec une majuscule chaque fois qu'il ne s'agit pas de la physique scolaire. On parler ainsi de la Physique académique, de la Physique dans la société...

6 Une unité de sens est un ensemble de mots correspondant à une information en rapport avec la question, par exemple, la réponse «j’ai appris la mécanique et l'électricité» comporte pour notre analyse deux unités de sens.

7 Par exemple, les variables «Savoirs cités de manière précise ", "Attentes de compréhension du monde ", "Présence en cours par obligation" regroupent respectivement le nombre d'unité de sens où un savoir ou savoir faire est cité de manière précise pour la première, le nombre 
d'unité de sens où l'élève exprime qu'il attend des savoirs de comprendre le monde, de l'expliquer, de le rendre intelligible etc. pour la seconde, et enfin le nombre d'unité de sens dans lesquelles l'élève exprime sa présence au cours de physique par obligation pour la troisième.

8 Il n'y a pas eu d'analyse des pratiques langagières: d'une part, les différences dans la rédaction d'élèves ayant quatre ans d'écart ne sont pas uniquement significatives de différences entre les rapports aux savoirs de la physique, d'autres part, les textes étaient généralement courts, proposant une réponse quasiment directe à la question posée, et donc offraient de ce fait peu de prise à l'analyse. Bautier et Rochex (1998, p. 122) voient dans des réponses courtes de certains lycéens, une manière de procéder identique à celle des élèves faibles de fin de collège ( 9 ème année de formation) qui, incapables de répondre globalement, répondent au plus près de la question posée. Cela n'est pas le cas ici, certains élèves étant pour le moins brillants. Peut-être peut-on attribuer cela au contexte de rédaction, dans le cadre d'un cours de physique, où traditionnellement, on répond précisément et brièvement aux questions posées.

9 Logiciel SPSS pour Windows

10 Le nombre d'éléments dans les idéal-types est variable car lié au nombre de variables dont les modalités sont significatives.

11 Un élève mobilisé «sun la physique trouve important le fait d'aller en cours de physique, un élève mobilisé «en" physique trouve important le fait d'apprendre les savoirs de la physique. Cette distinction est faite par Charlot et al. (1992, p. 71) à propos de la mobilisation à l'école et sur l'école.

12 Il n'y a pas eu d'analyse langagière des bilans de savoir scolaire. D’une part, les textes produits sont relativement courts, peut-être en raison de leur contexte de production (cf. note 8), et/ou parce qu'ils concernent uniquement le savoir scolaire. D'autre part, Bautier et Rochex (1998, p. 114) signalent la nécessité d'une pluralité de données pour établir des interprétations d'ordre cognitif à partir d'analyses linguistiques, ce dont nous ne disposions pas.

13 La disparité dans la taille des groupes n'est pas volontaire; elle reflète la plus ou moins grande difficulté à trouver, dans les quatre sections de départ, des élèves entretenant avec les savoirs de la physique un rapport très proche des différents idéal-types déjà construits.

14 Les raisons de cette importance, énoncées dans l'entretien, sont trop diverses pour être idéalisées.

15 Voir note 11.

16 On retrouve ces processus et phénomènes chez des élèves faibles des classes de fin de collège (Charlot et al., 1992, p. 148-150 et 76-77).

17 Pourtant, sur les cinq sujets les plus mobilisés en physique, quatre sont des garçons, alors que nous avons recherché systématiquement une parité dans le choix des individus.

18 Ces chercheurs distinguent les trois mêmes idéal-types en fonction de la manière dont les élèves qui y sont associés considèrent le lycée: le lycée comme une fin en soi, comme un lieu stratégique pour obtenir un métier et un statut social, comme un lieu de transformation de soi.

19 Cette remarque a été faite pour les sciences en général par exemple par Osborne et Collins (2000) et Munro et Elsom (2000). 


\section{Bibliographie}

Bautier, E. \& Rochex, J.-Y. (1998). L'expérience scolaire des nouveaux lycéens. Démocratisation ou massification? Paris: Armand Colin.

Caillot, M. (2001). Y a-t-il des élèves en didactique des sciences? Ou quelles références pour l'élève. In A. Terrisse, (Ed.) Didactique des disciplines, les références au savoir (pp. 141-155). Bruxelles: De Boeck.

Catel, L, Coquidé, M.-L. \& Gallezot, M. (2002). Rapport au savoir et apprentissage différencié de savoirs scientifiques de collégiens et de lycéens: quelles questions. Aster, 35, 123-148.

Charlot, B. (1997). Rapport au savoir: Eléments pour une théorie. Anthropos: Paris

Charlot, B. (1999a). Le rapport au savoir. In J . Bourdon \& Cl. Thélot (Dir.), Education et formation: l'apport de la recherche aux politiques éducatives (pp. 17-34). Paris: Editions du CNRS.

Charlot, B. (1999b). Le rapport au savoir en milieu populaire une recherche dans les lycées professionnels de banlieue. Paris: Anthropos.

Charlot, B., Bautier, E. \& Rochex, J.Y. (1992). Ecole et savoir dans les banlieues et ailleurs. Paris: Armand Colin.

Coll, R.K., Dalgety, J. \& Salter, D. (2002). The development of the chemistry attitude and experiences questionnaires (CAEQ). Chemistry Education Research and Practice in Europe, 3, (1), 19-32. Online: [http://www.uoi.gr/cerp/2002_February/pdfl04Coll.pdf].

Cosnefroy, L. (2004). Note de synthèse: Apprendre, faire mieux que les autres, éviter l'échec: l'influence de l'orientation des buts sur les apprentissages scolaires. Revue Française de Pédagogie, 147, 107-128.

Ebenezer, J.V. \& Zoller, U. (1993). Grade 10 student's perception of and attitudes toward science teaching and school science. Journal of Research in Science Teaching, 30, (2), 175-186.

Gardner, P.L. (1975). Attitudes to science: a review. Studies in Science Education, 2, 3-41.

Gardner, P.L. (1995). Measuring attitudes to science: unidimensionality and internal consistency revisited. Research in Science Education, 25, (3), 283-289.

Hanrahan, M. (1998). The effect of learning environment factors on students' motivation and learning. International Journal of Science Education, 20, (6), 737-753.

Havard, N. (1996). Student attitudes to studying A-level sciences. Public Understanding of Science, 5, (4), 321-330.

Hendley D., Stables, S. \& Stables A. (1996). Pupil's subject preference at key stage 3 in South Wales. Educational Studies, 22, (2), 177-186.

Meece, J. (1991). The classroom context and student's motivational goals. In M.L. Maher \& P.R. Pintrich (Ed.), Advances in Motivation and Achievement, 7, 261-286. Greenwich, CT: JAI Press.

Munro, M. \& Elsom, D. (2000). Choosing Science at 16: the Influences of Science Teachers and Careers Advisers on Students' Decisions about Science Subjects and Sciences Technology Careers. (NICEC Research Report). Cambridge: Careers Research and Advisor Centre. Online: [http://www.crac.org.uk/nicec/publications/pdfs/choosing_science.pdf].

Oppenheim, A.N. (1992). Questionnaire, Design, Interviewing and Attitude Measurement. (London: Continuum.

Osborne, J. \& Collins, S. (2000). Pupil's and Parent's Views of the School Science Curriculum. London: King's College.

Osborne, J., Simon, S. \& Collins, S. (2003). Attitude toward science a review of litterature and its implications. International Journal of Science Education, 25, (9), 1049-1079.

Piburn, M.D. \& Baker, D.R. (1993). If I were a teacher... qualitative study of attitude towards science. Science Education, 77, (4), 393-406.

Porchet, M. (2002). Les jeunes et les études scientifiques: les raisons de la désaffection - un plan d'action. Rapport à l'attention du Ministre de l'Education Nationale. Online: [www.education.gouv.fr/rapport/porchet.pdf]. 
Reid, N. \& Skryabina, E.A. (2002). Attitudes towards physics. Research in Science \& Technological Education, 20, (1), 67-81.

Schibeci, R.A. (1984). Attitude to science: an update. Studies in Science Education, 11, 26-59.

Venturini, P. (2005). Rapports idéal-typiques à la physique d'élèves de l'enseignement secondaire. Didaskalia, 26. 9-32

Venturini, P. (2004a). Note de synthèse: Attitudes des élèves envers les sciences: le point des recherches. Revue Française de Pédagogie, 149, 97-121.

Venturini, P. (2004b). Relations entre les rapports entretenus par des élèves de Troisième avec le savoir scolaire et avec les savoirs de la physique. In actes du Communication orale avec actes au $5^{\text {ième }}$ congrès international "Actualité de la recherche en éducation et en formation". (CNAM, Paris, 31 août-4 septembre 2004). Online: [http://www.aecse.net].

Venturini, P, \& Albe, V. (2002). Interprétation des similitudes et différences dans la maîtrise conceptuelle d'étudiants en électromagnétisme à partir de leur(s) rapport(s) au(x) savoir(s). Aster, 35, 165-188.

Weber, M. (1965). Essais sur la théorie des sciences. Paris: Plon.

Weingurgh, M.H. (1995). Gender differences in student attitudes towards science: a metaanalysis of the literature from 1970 to 1991. Journal of Research in Science Teaching, 32, (4), 387-398.

Mots clés: rapport aux savoirs de la physique; enseignement de la physique; enseignement secondaire; didactique de la physique; implication de l'élève

\section{Phänomene und Prozesse, die die Ansehung von physikalischem Wissen beeinflussen: Fallstudie über französische Schüler in der zehnten Klasse}

\section{Zusammenfassung}

Dieser Artikel beschäftigt sich mit der Frage nach der Beteiligung von Schülern an Physikprojekten und $\neg$-arbeiten in der Schule. Diese Frage, welche die angelsächsische Forschung dazu brachte, eine große Anzahl Studien über die Einstellung zur Wissenschaft durchzuführen, wird im Artikel dahingehend bearbeitet, dass die Ansehung bzw. Relation voEinstellung zum Wissen als theoretischer Rahmen benutzt wird. Aufbauend auf früheren Arbeiten werden fünf idealtypische Relationen von physikalischem Fachwissen präsentiert, charakterisiert durch die Natur der Aktivierung die wir mit jeder in Verbindung bringen können. Diese Studie führt die Arbeit weiter und hat zu einer Identifizierung von bestimmten Phänomenen und Prozessen geführt, die diese Relationen von Wissen beeinflussen. Die Phänomene betreffen den jeweiligen Schulkontext, Ideen über Physik und berufliche Zukunftspläne. Weiterhin hat sie uns erlaubt, die Verbindung zwischen Relationen von physikalischem und schulischem Wissen zu untersuchen.

Schlagworte: Beziehung zu physikalischem Wissen, Physikunterricht, Unterricht in der Sekundarstufe, Didaktik der Physik, Beteiligung des Schülers 


\title{
Fenomeni e processo che incidono sul rapporto col sapere nella fisica: il caso degli allievi francesi al decimo anno di formazione
}

\section{Riassunto}

Questo contributo discute il coinvolgimento degli allievi nel loro apprendimento scolastico della fisica. La questione, già trattata in molteplici studi anglosassoni sull'atteggiamento verso le scienze, viene affrontata utilizzando il rapporto con il sapere quale quadro di riferimento teorico. Alcuni lavori realizzati in precedenza avevano permesso di delineare una tipologia di cinque rapporti con il sapere nella fisica, caratterizzati dalla natura dell'attivazione del sapere. Lo studio attuale continua questo lavoro e permette di identificare una parte dei fenomeni e dei processi che intervengono in questi rapporti. Si tratta di fenomeni che riguardano l'ambiente scolastico, le rappresentazioni della fisica accademica e il progetto professionale. Inoltre si è potuta esaminare la relazione esistente tra i rapporti con il sapere nella fisica e i rapporti nei confronti del sapere scolastico in genere.

Parole chiave: relazione con i sapere in fisica, insegnamento della fisica, insegnamento secondario, didattica della fisica, coinvolgimento dell'allievo

\section{Phenomena and processes having effect on relation to physics knowledge: case of French pupils in grade 10}

\begin{abstract}
This article concerns the pupil's involvement in their physics school work. This question, which led Anglo-Saxon research to make a large number of studies about attitudes towards science, is envisaged here using relation to knowledge as a theoretical frame. A previous work had allowed us to built five ideal-typical relations to physics knowledge, characterized by the nature of the mobilisation that we can associate with each of them. This study carries on this work and had led to the identification of certain phenomena and processes which affect these relations to knowledge. The phenomena concern school context, ideas about physics, and professional plan. It has also allowed to examine the articulation between relations to physics knowledge and relations to academic knowledge.
\end{abstract}

Key words: relation to physics knowledge; physics teaching; secondary teaching; didactics of physics; student involvement 
